\title{
Experimental and clinical nephroprotection by the xanthine oxidase inhibitor febuxostat
}

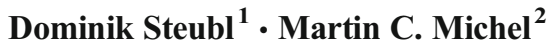

Received: 10 May 2016 / Accepted: 11 May 2016 / Published online: 25 May 2016

(C) Springer-Verlag Berlin Heidelberg 2016

Hyperuricemia is a common phenomenon in chronic kidney disease (CKD), most presumably due to reduced renal clearance of uric acid. Whether this is a secondary phenomenon that needs to be treated only in case of symptomatic gout or if hyperuricemia contributes to the progression of CKD is largely disputed. "Gouty nephropathy," focal interstitial urate crystal deposition causing chronic renal failure and eventually end-stage-renal-disease (ESRD), has become a rare disease (Gonick et al. 1965). Independently from this, data gathered from mouse models suggest that hyperuricemia may be a cause of CKD and its progression by causing renal arteriolopathy, tubular interstitial inflammation, and fibrosis as well as epithelial-to-mesenchymal transition (Kang et al. 2002; Sánchez-Lozada et al. 2005). Recent trials enforce the hypothesis that hyperuricemia is an independent risk factor for progression of CKD in both children/adolescents and grownups (Rodenbach et al. 2015; Li et al. 2014), although this has been put into question by other studies on large CKD cohorts, in which no association between uric acid levels and CKD progression was found (Sturm et al. 2008; Madero et al. 2009).

Despite this conflicting data, it has been advocated by many authors that pharmacological treatment of hyperuricemia in CKD patients using allopurinol might slow down CKD progression (Siu et al. 2006; Goicoechea et al. 2010, 2015; Kanji et al. 2015). This might not only be due to its

\section{Dominik Steubl}

Dominik.steubl@1rz.tum.de

1 Department of Nephrology, Klinikum rechts der Isar, Technische Universität München, Ismaninger Str. 22, 81675 Munich, Germany

2 Department of Pharmacology, Johannes Gutenberg University, Mainz, Germany urate-lowering capacities but also by reducing radical oxygen species, the latter being produced by xanthine oxidase, the therapeutic target of allopurinol (Kang and Chen 2011). Febuxostat has been introduced as a new, non-purine, and selective xanthine oxidase inhibitor and, therefore, uric acid-lowering agent. Besides its usual indication in patients with recurrent gout it might, similar to allopurinol as mentioned above, be used in CKD to ameliorate kidney function decline. In basic research, febuxostat prevented arteriolopathy and decline of renal function in the classic model of 5/6 nephrectomized rats independently from hyperuricemia (Sánchez-Lozada et al. 2008a). The same authors stated that febuxostat improved glomerular hemodynamics and vascular morphology in rat models of fructose-induced metabolic syndrome and experimental hyperuricemia (Sánchez-Lozada et al. 2008b, c). This finding in $5 / 6$ nephrectomized rats was reproduced in several models of renal inflammation: in two rat models of unilateral ureteral obstructive nephropathy, febuxostat treatment was associated with less interstitial fibrosis and inflammation (Omori et al. 2012; Cao et al. 2015), an observation that is in line with the results of Tsuda et al. gathered from a ischemia-reperfusion-injury rat model (Tsuda et al. 2012). Lee et al. evaluated febuxostat in a streptozotocin-induced diabetic rat model and also found less expression of proinflammatory genes in diabetic rats treated with febuxostat and even positive effects on albuminuria (Lee et al. 2014). Evaluating albuminuria as a surrogate parameter for kidney function and glomerular integrity, febuxostat was associated with reduced albuminuria, in a rat model of diabetic nephropathy, but this did not exhibit dose dependency (Nakamura et al. 2016). Furthermore, despite the fact that uric acid has been advocated to play a role in the development of cardiovascular disease and hypertension, no positive effect of febuxostat 
on blood pressure levels were detected in hypertensive rates (Szasz et al. 2013).

An article in this issue of Naunyn-Schmiedeberg's Archives of Pharmacology describes prevention of cisplatininduced renal damage in rats by febuxostat (Fahmi et al. 2016a). The authors have evaluated the influence of different oral febuxostat dosages on kidney function in rats treated with a nephrotoxic dose of intraperitoneally administered cisplatin. While not lowering uric acid, febuxostat prevented acute kidney injury in this model. Possibly, the antiinflammatory effect of febuxostat appears to be organ independent, as the authors already showed similar protective effects on lung tissue in a rat model previously (Fahmi et al. 2016b). While some antioxidant effect was observed, the nephroprotection occurred at least partly at a dose exhibiting limited antioxidant effects. These data extend the range of nephropathy models in which febuxostat is effective as a preventive or treatment agent; they also raise questions on the role of xanthine oxidase and antioxidant effects in such nephroprotection.

Transferring these results into clinical practice, a couple of trials evaluated febuxostat in view of its capacity to slow down progression of CKD: in a prospective, randomized, and placebo-controlled trial, febuxostat lead to an increase of estimated glomerular filtration rate (eGFR) in a time period of 6 months in CKD 3-4, compared to a significant deterioration of eGFR in placebo-treated patients over the same time (Sircar et al. 2015).

Whether and to what extent the renoprotective effects of febuxostat may outperform those of allopurinol has not been studied well until now. A small prospective, randomized, open-label trial that compared both drugs in CKD patients over a time period of 12 weeks showed a stronger reduction of uric acid serum levels and even proteinuria, but no difference in change of eGFR was seen (Tanaka et al. 2015). However, the latter fact probably needs to be attributed to the short follow-up period. In a bigger but retrospectively designed cohort study, patients that were switched from allopurinol to febuxostat and then followed over 1 year had a lower decrease of eGFR and a higher decrease of uric acid serum levels at the end of follow-up compared to a group of patients maintained on allopurinol (Tsuruta et al. 2014). In patients undergoing cardiac surgery, febuxostat showed better renoprotective and antioxidative effects compared to allopurinol (Sezai et al. 2015). Another non-interventional, retrospective study proved that febuxostat can be safely used in CKD 3-5 patients; furthermore, it was also successful in this advanced CKD stages in terms of eGFR preservation (Shibagaki et al. 2014). Taken together, febuxostat appears to be a promising tool to slow down the progression of CKD in patients with various disease entities. However, further prospective clinical studies are needed to confirm these data.

\section{References}

Gonick HC, Rubini MD, Gleason IO, Sommers SC (1965) The renal lesion in gout. Ann Intern Med 62:667-674

Kang DH, Nakagawa T, Feng L, Watanabe S, Han L, Mazzali M, Truong L, Harris R, Johnson RJ (2002) A role for uric acid in the progression of renal disease. J Am Soc Nephrol 13:2888-2897

Sánchez-Lozada LG, Tapia E, Santamaría J, Avila-Casado C, Soto V, Nepomuceno T, Rodríguez-Iturbe B, Johnson RJ, Herrera-Acosta J (2005) Mild hyperuricemia induces vasoconstriction and maintains glomerular hypertension in normal and remnant kidney rats. Kidney Int 67:237-247

Rodenbach KE, Schneider MF, Furth SL, Moxey-Mims MM, Mitsnefes MM, Weaver DJ, Warady BA, Schwartz GJ (2015) Hyperuricemia and progression of CKD in children and adolescents: the chronic kidney disease in children (CKiD) cohort study. Am J Kidney Dis 66:984-992

Li L, Yang C, Zhao Y, Zeng X, Liu F, Fu P (2014) Is hyperuricemia an independent risk factor for new-onset chronic kidney disease?: a systematic review and meta-analysis based on observational cohort studies. BMC Nephrol 15:122

Sturm G, Kollerits B, Neyer U, Ritz E, Kronenberg F, MMKD Study Group (2008) Uric acid as a risk factor for progression of nondiabetic chronic kidney disease? The mild to moderate kidney disease (MMKD) study. Exp Gerontol 43:347-532

Madero M, Sarnak MJ, Wang X, et al. (2009) Uric acid and long-term outcomes in CKD. Am J Kidney Dis 53:796-803

Siu YP, Leung KT, Tong MK, Kwan TH (2006) Use of allopurinol in slowing the progression of renal disease through its ability to lower serum uric acid level. Am J Kidney Dis 47:51-59

Goicoechea M, de Vinuesa SG, Verdalles U, et al. (2010) Effect of allopurinol in chronic kidney disease progression and cardiovascular risk. Clin J Am Soc Nephrol 5:1388-1393

Goicoechea M, Garcia de Vinuesa S, Verdalles U, et al. (2015) Allopurinol and progression of CKD and cardiovascular events: long-term follow-up of a randomized clinical trial. Am J Kidney Dis 65:543-549

Kanji T, Gandhi M, Clase CM, Yang R (2015) Urate lowering therapy to improve renal outcomes in patients with chronic kidney disease: systematic review and meta-analysis. BMC Nephrol 16:58

Kang DH, Chen W (2011) Uric acid and chronic kidney disease: new understanding of an old problem. Semin Nephrol 31:447-552

Sánchez-Lozada LG, Tapia E, Soto V, Avila-Casado C, Franco M, Wessale JL, Zhao L, Johnson RJ (2008a) Effect of febuxostat on the progression of renal disease in 5/6 nephrectomy rats with and without hyperuricemia. Nephron Physiol 108:69-78

Sánchez-Lozada LG, Tapia E, Bautista-García P, Soto V, Avila-Casado C, Vega-Campos IP, Nakagawa T, Zhao L, Franco M, Johnson RJ (2008b) Effects of febuxostat on metabolic and renal alterations in rats with fructose-induced metabolic syndrome. Am J Physiol Ren Physiol 294:F710-F718

Sánchez-Lozada LG, Tapia E, Soto V, Avila-Casado C, Franco M, Zhao L, Johnson RJ (2008c) Treatment with the xanthine oxidase inhibitor febuxostat lowers uric acid and alleviates systemic and glomerular hypertension in experimental hyperuricaemia. Nephrol Dial Transplant 23:1179-1185

Omori H, Kawada N, Inoue K, Ueda Y, Yamamoto R, Matsui I, Kaimori J, Takabatake Y, Moriyama T, Isaka Y, Rakugi H (2012) Use of xanthine oxidase inhibitor febuxostat inhibits renal interstitial inflammation and fibrosis in unilateral ureteral obstructive nephropathy. Clin Exp Nephrol 16:549-556

Cao J, Li Y, Peng Y, Zhang Y, Li H, Li R, Xia A (2015) Febuxostat prevents renal interstitial fibrosis by the activation of BMP-7 signaling and inhibition of USAG-1 expression in rats. Am J Nephrol 42: 369-378 
Tsuda H, Kawada N, Kaimori JY, Kitamura H, Moriyama T, Rakugi H, Takahara S, Isaka Y (2012) Febuxostat suppressed renal ischemiareperfusion injury via reduced oxidative stress. Biochem Biophys Res Commun 427:266-272

Lee HJ, Jeong KH, Kim YG, Moon JY, Lee SH, Ihm CG, Sung JY, Lee TW (2014) Febuxostat ameliorates diabetic renal injury in a streptozotocin-induced diabetic rat model. Am J Nephrol 40:56-63

Nakamura T, Murase T, Nampei M, Morimoto N, Ashizawa N, Iwanaga T, Sakamoto R (2016) Effects of topiroxostat and febuxostat on urinary albumin excretion and plasma xanthine oxidoreductase activity in $\mathrm{db} /$ db mice. Eur J Pharmacol. doi:10.1016/j.ejphar.2016.03.055

Szasz T, Davis RP, Garver HS, Burnett RJ, Fink GD, Watts SW (2013) Long-term inhibition of xanthine oxidase by febuxostat does not decrease blood pressure in deoxycorticosterone acetate (DOCA)-salt hypertensive rats. PLoS One 8:e56046

Fahmi AN, Shehatou GS, Shebl AM, Salem HA (2016a) Febuxostat exerts dose-dependent renoprotection in rats with cisplatin-induced acute renal injury. Naunyn Schmiedebergs Arch Pharmacol. doi:10. 1007/s00210-016-1258-y

Fahmi AN, Shehatou GS, Shebl AM, Salem HA (2016b) Febuxostat protects rats against lipopolysaccharide-induced lung inflammation in a dose-dependent manner. Naunyn Schmiedeberg's Arch Pharmacol 389:269-278
Sircar D, Chatterjee S, Waikhom R, Golay V, Raychaudhury A, Chatterjee S, Pandey R (2015) Efficacy of febuxostat for slowing the GFR decline in patients with CKD and asymptomatic hyperuricemia: a 6-month, double-blind, randomized placebo-controlled trial. Am J Kidney Dis 66:945-950

Tanaka K, Nakayama M, Kanno M, Kimura H, Watanabe K, Tani Y, Hayashi Y, Asahi K, Terawaki H, Watanabe T (2015) Renoprotective effects of febuxostat in hyperuricemic patients with chronic kidney disease: a parallel-group, randomized, controlled trial. Clin Exp Nephrol 19:1044-1053

Tsuruta Y, Mochizuki T, Moriyama T, Itabashi M, Takei T, Tsuchiya K, Nitta K (2014) Switching from allopurinol to febuxostat for the treatment of hyperuricemia and renal function in patients with chronic kidney disease. Clin Rheumatol 33:1643-1648

Sezai A, Soma M, Nakata K, Osaka S, Ishii Y, Yaoita H, Hata H, Shiono M (2015) Comparison of febuxostat and allopurinol for hyperuricemia in cardiac surgery patients with chronic kidney disease (NUFLASH trial for CKD). J Cardiol 66:298-303

Shibagaki Y, Ohno I, Hosoya T, Kimura K (2014) Safety, efficacy and renal effect of febuxostat in patients with moderate-to-severe kidney dysfunction. Hypertens Res 37:919-925 